\title{
FIBREWISE LOCALIZATION AND COMPLETION
}

\author{
BY
}

J. P. MAY

\begin{abstract}
The behavior of fibrewise localization and completion on the classifying space level is analyzed. The relationship of these constructions to fibrewise joins and smash products and to orientations of spherical fibrations is also analyzed. This theory is essential to validate Sullivan's proof of the Adams conjecture.
\end{abstract}

In Sullivan's beautiful proof of the Adams conjecture [20], perhaps the crucial technical point is the behavior on the classifying space level of fibrewise localization and completion. The idea is that, for a spherical fibration $S^{n} \rightarrow E \rightarrow B$, one can construct new fibrations

$$
S_{T}^{n} \rightarrow E_{T}^{f} \rightarrow B \quad \text { and } \quad \hat{S}_{T}^{n} \rightarrow \hat{E}_{T}^{f} \rightarrow B .
$$

Here $T$ denotes a set of primes, $X_{T}$ and $\hat{X}_{T}$ denote the localization and completion of a (nilpotent) space $X$ at $T$, and $E_{T}^{f}$ and $\hat{E}_{T}^{f}$ denote "fibrewise" localized and completed total spaces. This procedure gives the following diagram of representable functors on the category $\mathcal{W}$ of spaces of the homotopy type of CW-complexes:

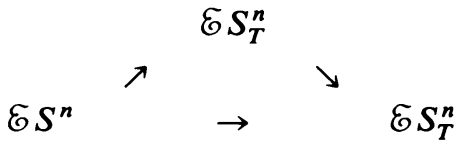

Here $\mathcal{E} X(B)$ is the set of equivalence classes of fibrations with fibre $X$ over a space $B \in \mathcal{W}$. There results a corresponding diagram of classifying spaces, and the point is to analyze its behavior in terms of localization and completion.

One can construct fibrewise localizations and completions in at least four ways. Sullivan [20] takes $B$ to be a simplicial complex and proceeds cell by cell. Bousfield and Kan [3] work with simplicial sets and give a nice functorial construction. It is also possible to work with Postnikov towers and proceed cocell by cocell [16]. None of these procedures bears any obvious relationship to behavior on the classifying space level. The question is not considered by Bousfield and Kan. I do not know whether or not Sullivan's outline of a proof of the relationship could be made rigorous. Certainly any such argument would be extremely long and technical.

We shall perform certain natural constructions on the classifying space level. These will give sufficient functoriality (in the variable $X$ ) on the classifying spaces for $\mathcal{E} X$ as to allow a new construction of fibrewise localizations and completions

Received by the editors February 23, 1979.

AMS (MOS) subject classifications (1970). Primary 55E50, 55F05, 55F15.

Key words and phrases. Adams conjecture, classifying space, localization, completion, fibre homotopy equivalence, fibrewise join, fibrewise smash product, orientation. 
by direct appeal to the classification theorem. With this approach, the behavior of these constructions on the classifying space level will be tautologically obvious.

We begin in $\$ 1$ with a review of classification theory for fibrations. We consider two basic variants, with based and unbased fibres, and we discuss fibrewise joins, smash products, and suspensions. In $\$ 2$, we compare functors and give a general treatment of orientation theory. In $\$ 3$, we study the naturality of classification theory with respect to changes of fibre. We specialize this study to the analysis of fibrewise localization and completion in $\$ 4$ and specialize further to spherical fibrations in $\$ 5$.

While our only concern has been to set up a rigorous theory, the interest naturally lies in the applications. The most important is Sullivan's proof of the Adams conjecture [20], his being the only argument which yields the much deeper unstable version of that result. Adams' initial step, the Dold theorem $\bmod k$ [1], is an obvious consequence of the present theory (Proposition 5.5). Various authors, such as Kahn [12] and Glover and Mislin [8], have used fibrewise localization to study manifolds.

In sum, this is a sufficiently powerful tool that I felt a full development to be imperative. The material of this paper largely dates to 1974 and was promised in [14], which is our basic source for classification theory.

It is a pleasure to thank Pete Bousfield for Proposition 4.2.

1. A review of classification theory. Let $X$ be a nondegenerately based space of the homotopy iype of a CW-complex. Define functors $\mathcal{E} X$ and $\mathcal{E}_{0} X$ on $\mathscr{W}$ as follows. For $B \in \mathcal{Q}, \varepsilon X(B)$ is the set of equivalence classes of Hurewicz fibrations over $B$, with fibres weakly homotopy equivalent to $X$, under the equivalence relation generated by those fibrewise maps over $B$ which restrict to weak equivalences on fibres. Define $\varepsilon_{0} X$ similarly, except restricting to fibrations with based fibres and to fibrewise maps which preserve the basepoints of fibres, these basepoints being required to specify together a section which is a fibrewise cofibration (see [14, 5.2]). For technical reasons, specifically the convenience of particular universal fibrations whose fibres need not be the homotopy type of $\mathrm{CW}$-complexes, these are our preferred definitions. However, we have the following observation.

LeMMA 1.1. $\mathcal{E} X(B)$ is equal to the set $\mathcal{E}^{\prime} X(B)$ of fibre homotopy equivalence classes of fibrations over $B$ with fibres homotopy equivalent to $X$, and similarly for $\mathcal{E}_{0} X(B)$.

Proof. $\mathcal{E}^{\prime} X(A)$ is a well-defined set in view of its natural injection to $\mathscr{E} X(A)$ and the classification theorem (1.2 below) for the latter, injectivity being a direct consequence of Dold's theorem [5, 6.3]; $[14,2.6]$. We must show surjectivity. That is, we must show that any fibration $\xi: E \rightarrow B$ with fibres weakly equivalent to $X$ is weakly equivalent to a fibration $\zeta: D \rightarrow B$ with fibres homotopy equivalent to $X$. The geometric realization of the total singular map $S \xi: S E \rightarrow S B$ maps to $\xi$ : 


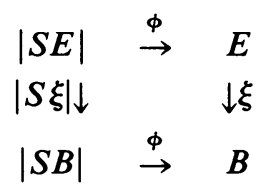

Replace $|S \xi|$ by a fibration $\xi^{\prime}: E^{\prime} \rightarrow|S B|$ in the usual way. The fibres of $\xi^{\prime}$ are of the homotopy type of CW-complexes because $E^{\prime}$ is so [18], [19]. Replacing $\xi$ by a fibration does not change its fibre homotopy type, hence we obtain a diagram as above with $|S E|$ replaced by $E^{\prime}$. By the five lemma, the resulting maps on fibres are weak equivalences, hence the fibres of $\xi^{\prime}$ are all homotopy equivalent to $X$. Since $B \in$ W $, \phi:|S B| \rightarrow B$ is a homotopy equivalence, say with homotopy inverse $\psi$. Let $\zeta: D \rightarrow B$ be $\psi^{-1}\left(\xi^{\prime}\right)$. It is easy to use the $C H P$ to construct a weak equivalence of fibrations from $\zeta$ to $\xi$.

Define $G X$ and $F X$ to be the topological monoid of homotopy equivalences of $X$ and its submonoid of based homotopy equivalences. Define $S G X$ and $S F X$ to be the respective submonoids of maps homotopic to the identity. Actually, since the identity map of $X$ need not be a nondegenerate basepoint for $H X$ in general, we should really understand $H X$ to be the monoid obtained by growing a whisker from the identity map (see [13, A.8] and [14, 9.3]). If $X$ is compact, but not in general otherwise, then $H X$ has the homotopy type of a CW-complex [17].

Let $B H X$ be the standard classifying space of $H X$ (e.g. $[14, \S 7])$. The following classification theorem is proven in $[14, \S 9]$. Let $\left[B, B^{\prime}\right]$ denote the set of homotopy classes of (unbased) maps $B \rightarrow B^{\prime}$.

THEOREM 1.2. There are natural bijections

$$
\mathcal{E} X(B) \cong[B, B G X] \text { and } \mathcal{E}_{0} X(B) \cong[B, B F X]
$$

When $X$ is a finite CW-complex, the classification of $\mathscr{E} X$ is due to Stasheff [19]. For general $X$, Allaud [2] and Dold [6] used Brown's representability theorem to classify $\mathcal{E} X$ (but see [14, p. vi]). Allaud also gave an argument which, together with $[14,7.7]$, implies that his classifying space is weakly equivalent to $B G X$. However, several of the results below depend on use of the particular universal fibration of $[14, \S 9]$, and its applicability depends on our precise definition of $\mathscr{E} X(B)$ and the concomitant freedom to exploit weak equivalences of fibrations and spaces not of the homotopy type of CW-complexes.

In view of Theorem 1.2, we define

$$
S \mathcal{E} X(B)=[B, B S G X] \text { and } S \mathcal{E}_{0} X(B)=[B, B S F X]
$$

As explained in [14, 10.4], these functors may be interpreted in terms of fibrations together with "reductions of their structural monoids". An orientation theoretic interpretation for appropriate $X$ will be given in the next section.

Fibrewise join defines a natural transformation $\mathcal{E} X(B) \times \mathcal{E} X\left(B^{\prime}\right) \rightarrow \mathcal{E} X(B \times$ $\left.B^{\prime}\right)$. This is an external operation, the corresponding internal operation being induced by the diagonal $\Delta^{*}: \mathcal{E} X(B \times B) \rightarrow \mathcal{E} X(B)$. Similarly, there is a fibrewise smash product $\varepsilon_{0} X(B) \times \mathcal{E}_{0} X\left(B^{\prime}\right) \rightarrow \varepsilon_{0} X\left(B \times B^{\prime}\right)$, the corresponding internal operation being written $\oplus$ and thought of as a Whitney sum [14, 5.6]. These 
constructions are simple because join and smash products are continuous functors (in contrast to localization and completion; see §4). For the same reason, join and smash product of maps specify continuous monoid homomorphisms

$$
*: G X \times G X^{\prime} \rightarrow G\left(X * X^{\prime}\right) \text { and } \wedge: F X \times F X^{\prime} \rightarrow F\left(X \wedge X^{\prime}\right) \text {. }
$$

Since the functor $B$ commutes with products, there result

$$
B(*): B G X \times B G X^{\prime} \rightarrow B G\left(X * X^{\prime}\right) \text { and } B(\wedge): B F X \times B F X^{\prime} \rightarrow B F\left(X \wedge X^{\prime}\right) \text {. }
$$

It is not hard to verify that $B(*)$ and $B(\wedge)$ represent the fibrewise join and smash product. One need only check that the pullbacks of the respective universal fibrations under $B(*)$ and $B(\wedge)$ are weakly equivalent to the fibrewise joins and smash products of the relevant universal fibrations. See Lewis [4, Chapter XI] for details in the (harder) smash product case.

The unreduced suspension $\Sigma^{+} X$ is the join $X * S^{0}$, hence fibrewise join with the trivial fibration $B \times S^{0} \rightarrow B$ specifies a fibrewise suspension operation $\mathscr{E} X(B) \rightarrow$ $\mathcal{E} \Sigma^{+} X(B)$. This is represented by the map $B G X \rightarrow B G \Sigma^{+} X$ induced by suspension of homotopy equivalences. Of course, with one of the cone points as basepoint, this map factors through $B F \Sigma^{+} X$. Similarly, the reduced suspension $\Sigma X$ is the smash product $X \wedge S^{1}$, hence fibrewise smash product with the trivial fibration $B \times S^{1}$ $\rightarrow B$ specifies $\mathcal{E}_{0} X(B) \rightarrow \mathcal{E}_{0} \Sigma X(B)$. This fibrewise suspension is represented by the evident map $B F X \rightarrow B F \Sigma X$.

Of course, we may also replace $G$ and $F$ by $S G$ and $S F$ throughout the above two paragraphs.

2. Comparisons of functors and orientation theory. We shall study our various represented functors by direct homotopical analysis of their classifying spaces. In this analysis, maps and diagrams are to be interpreted in the category obtained from the homotopy category of compactly generated spaces by formally inverting weak equivalences (or, equivalently, by passing to $\mathrm{CW}$-approximations). This is a reasonable place to work on the classifying space level since a weak equivalence $Y \rightarrow Z$ induces a bijection $[B, Y] \rightarrow[B, Z]$ for $B \in \mathcal{W}$, by Whitehead's theorem.

For example, let us compare the two kinds of fibrewise suspension just defined. The quotient map $\pi: \Sigma^{+} X \rightarrow \Sigma X$ is a (based) homotopy equivalence. By [14, §12], or its generalization in the following section, $\pi$ induces compatible weak equivalences $\pi_{\#}: B H \Sigma^{+} X \rightarrow B H \Sigma X$ (for $H=G, F$, etc.) such that the following diagram commutes, where $j: F X \rightarrow G X$ is the inclusion.

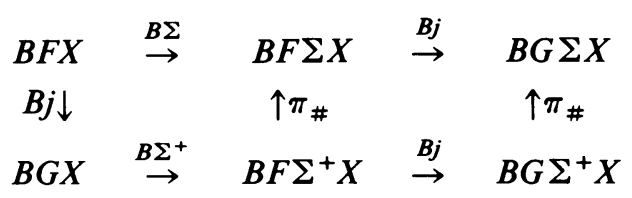

This implies the compatibility of the unreduced and reduced fibrewise suspensions under the natural forgetful map $\mathcal{E}_{0} X(B) \rightarrow \mathcal{E} X(B)$. Indeed, as a matter of definition, $\mathscr{E} X$ depends only on the homotopy type of $X$; and the transformation represented by $\lambda_{\#}$ for a homotopy equivalence $\lambda: X \rightarrow X^{\prime}$ is the identity by inspection of the constructions involved. Precisely, $\lambda_{\#}$ is really a formal composite 
$B F X \rightarrow D \leftarrow B F X^{\prime}$ of weak equivalences with an intermediate space $D$, and the universal fibrations over $B F X$ and $B F X^{\prime}$ are weakly equivalent to pullbacks of one and the same fibration over $D$, necessarily also universal. Thus the diagram above interprets as follows on the level of represented functors.

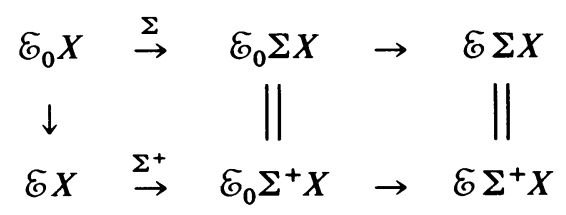

Of course, we could instead have obtained this comparison directly by considering the natural collapsing map from fibrewise joins with $B \times S^{0} \rightarrow B$ to fibrewise smash products with $B \times S^{1} \rightarrow B$, but we shall later be applying similar classifying space level arguments to situations in which no such elementary fibration level arguments are available.

The inclusions $j$ above fit into the following comparison of the spaces $B H X$, the maps $i$ also being inclusions.

Proposition 2.1. There is a commutative diagram

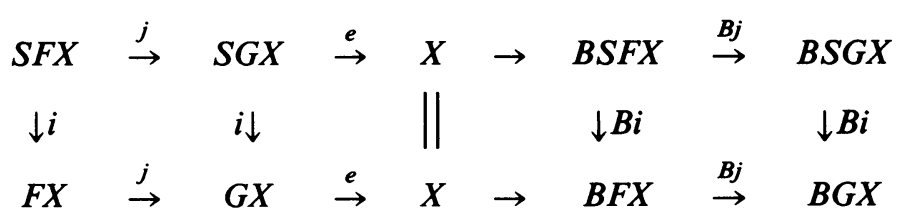

in which the rows are fibration sequences, the maps Bi are homotopy universal covers, and the left and right squares are pullbacks.

Proof. The assertion about $B i$ is immediate from a comparison of universal principal quasifibrations. The left square is trivially a pullback and implies that the right square is a pullback by $[13,11.6]$. The evaluation map $e: G X \rightarrow X$ is a fibration with fibre $F X$ since $X$ is nondegenerately based, and it remains to extend this fibration to the right (since the argument for the top row will be the same). Recall the two-sided bar construction from [14, §7] and observe that $e$ factors as a composite

$$
G X \stackrel{\tau}{\rightarrow} B(G X, F X, *) \stackrel{\varepsilon(e)}{\rightarrow} X
$$

(see $[13,9.2])$. That the bottom row is a fibration sequence follows directly from $[14,8.8]$, the diagram there and a comparison of fibrations giving that $\varepsilon(e)$ is a weak equivalence and the unlabelled arrow $X \rightarrow B F X$ being the (formal) composite of $\varepsilon(e)^{-1}$ and the natural quasifibration $B(G X, F X, *) \rightarrow B F X$.

The proposition immediately implies Gottlieb's result [9] that, up to weak equivalence, $B F X$ is the total space of the universal fibration over $B G X$.

Turning to orientation theory, fix a commutative ring $R$ and let $F R$ denote its group of units. We adopt the following definition. 
Definition 2.2. An $R$-fundamental class for $X$ is a map $i: S^{n} \rightarrow X$ such that $i^{*}$ : $\tilde{H}^{q}(X ; R) \rightarrow \tilde{H}^{q}\left(S^{n} ; R\right)$ is an isomorphism for all $q \leqslant n$ (so that $\tilde{H}^{n}(X ; R) \simeq R$ and $\tilde{H}^{q}(X ; R)=0$ for $\left.q<n\right)$ and the homomorphism $\nu: \pi_{0} F X \rightarrow F R$ specified by $f^{*}(\iota)=\nu(f) \iota$ is an isomorphism, where $\iota \in \tilde{H}^{n}(X ; R)$ is the inverse image of the canonical fundamental class in $\tilde{H}^{n}\left(S^{n} ; R\right)$.

Our main examples will be localized and completed spheres. We assume given $i$ and $\iota$ for $X$ in what follows.

Definition 2.3. Let $\xi: E \rightarrow B$ be a fibration with fibres weakly equivalent to $X$ and with a section which is a fibrewise cofibration. Define the Thom space of $\xi$ to be the quotient space $T \xi=E / B$. (See [15, p. 49] for discussion.) Define an orientation (or $R$-orientation) of $\xi$ to be a class $\mu \in \tilde{H}^{n}(T \xi ; R)$ such that $\mu$ restricts to a generator of the free $R$-module $\tilde{H}^{n}(T \chi ; R)$ for each fibre $\chi: \xi^{-1}(b) \rightarrow\{b\}$ of $\xi$.

Thus if $\phi: \xi^{-1}(b) \rightarrow X$ is a weak equivalence, then $\mu$ restricts to a unit of $R$ times $\phi^{*}(\iota)$. If $H^{q}(X ; R)=0$ for $q>n$, so that $X$ is an $R$-cohomology $n$-sphere, then the standard Serre spectral sequence argument gives that the cup product with $\mu$ specifies a Thom isomorphism $H^{*}(B ; R) \cong \tilde{H}^{*}(T \xi ; R)$. The facts about Thom spaces recorded in $[15$, p. 51$]$, such as

$$
T(\xi \wedge \psi) \cong T \xi \wedge T \psi \text { and } T(\xi \oplus \psi) \cong T \xi^{*}(\psi) / T \psi,
$$

remain valid in the present context. Moreover, the discussion of orientations of trivial fibrations, fibrewise smash products, etc., of [15, III.5.1] carries over with only trivial modifications.

Let $\mathcal{E}_{0}(X ; R)(B)$ denote the set of equivalence classes of pairs $(\xi, \mu)$, where $\xi$ is a fibration over $B$ with (based) fibres weakly equivalent to $X$ and $\mu$ is an orientation of $\xi$. The equivalence relation is that generated by orientation-preserving fibrewise weak equivalences.

THEOREM 2.4. There is a natural bijection

$$
S \mathcal{E}_{0} X(B) \cong \mathcal{E}_{0}(X ; R)(B)
$$

provided that $X$ has an $R$-fundamental class.

Proof. The monoid $F X$ acts on $F R$ via the homomorphism $\nu \delta: F X \rightarrow F R$, where $\delta: F X \rightarrow \pi_{0} F X$ is the discretization map and $\nu: \pi_{0} F X \rightarrow F R$ is the isomorphism of Definition 2.2. Thus we can form $B(F R, F X$, *). Since $S F X$ acts trivially on $F R$, we have a natural inclusion

$$
B S F X=B(*, S F X, *) \rightarrow B(F R, F X, *) .
$$

An evident comparison of quasifibrations over $B F X$ (compare Proposition 2.1) shows that this map is a weak equivalence. Thus it suffices to show that $B(F R, F X, *)$ classifies $\varepsilon_{0}(X ; R)$. Let $F(X, Z)$ denote the space of based maps $X \rightarrow Z$ and consider

$$
F(X, K(R, n)) \stackrel{i^{*}}{\rightarrow} F\left(S^{n}, K(R, n)\right)=\Omega^{n} K(R, n)=K(R, 0) \stackrel{\delta}{\rightarrow} R \supset F R .
$$

Our cohomological hypothesis on $i$ is precisely the assertion that $i^{*}$ here is a weak equivalence. Let $Y$ denote the union of those components of $F(X, K(R, n))$ which map to $F R$. Then $\delta i^{*}: Y \rightarrow F R$ is an $F X$-equivariant weak equivalence, where $F X$ 
acts on $Y$ by composition. Thus $B(Y, F X, *)$ is weakly equivalent to $B(F R, F X, *)$. But if $Z=K(R, n)$, then $(Y, Z)$ is an "admissible pair for $X \mathcal{T}$ " in the sense of [14, $10.2]$, by $[14,10.6]$ (with its compactness assumption deleted and $X \mathscr{V}$ there replaced by $X \mathcal{T}$ here to compensate; see [14, p. 29] for the categories $X \mathcal{T}$ and $X \mathscr{V})$. By $[14,11.1], B(Y, F X, *)$ classifies equivalence classes of $X$-fibrations with " $Y$-structure". The last notion is defined in [14, 10.1] and is easily checked to reduce to precisely the same thing as an orientation in the present context. Concretely, we have an evident quasifibration

$$
B(Y, F X, X) \rightarrow B(Y, F X, *)
$$

with section, and the evaluation map $Y \times X \rightarrow Z$ induces a map, or cohomology class,

$$
B(Y, F X, X) / B(Y, F X, *) \rightarrow K(R, n) .
$$

These data lead to the required universal oriented fibration over $B(Y, F X, *)$.

The homotopy universal cover $B S F X \rightarrow B F X$ represents the transformation $\mathcal{E}_{0}(X ; R) \rightarrow \mathcal{E}_{0} X$ given by neglect of orientations, and we have the following addendum.

COROllary 2.5. The (homotopical) action of $\pi_{1} B F X \cong F R$ on $B S F X$ given by covering transformations of the (actual) universal cover of $B F X$ represents the action of $F R$ on $\mathcal{E}_{0}(X ; R)(B)$ given by multiplication of orientations by units.

PROof. Covering transformations are determined by the action of $\pi_{1}$ on fibres, and the comparisons

$$
B S F X \rightarrow B(F R, F X, *) \leftarrow B(Y, F X, *)
$$

of spaces over $B F X$ reduce the question to consideration of the action of $\pi_{1} B F X$ on fibres of the fibration associated to the quasifibration $B(Y, F X, *) \rightarrow B F X$. For $f \in F X$, the loop $t \rightarrow|[f],(1-t, t)|$ in $B F X$ lifts to the map $Y \times I \rightarrow B(Y, F X, *)$ specified by $(y, t) \rightarrow|y[f],(1-t, t)|$. At $t=0$, this is the inclusion of the fibre over the standard basepoint $*=\mid[]$, (1)|; at $t=1$, this fibre has been translated by $f$. This description lifts to the associated fibration. Now $Y \simeq F R \subset F(X, K(R, n))$, and there is a unique homotopy class $g$ of self-equivalences of $K(R, n)$ such that $g_{*} \simeq f^{*}: Y \rightarrow Y$. Clearly $g$ acts on the quasifibration

$$
B(Y, F X, X) \rightarrow B(Y, F X, *)
$$

(by left composition on the level of simplicial spaces and passage to realization) and induces multiplication by the unit $g$ on its orientation. This statement lifts to the associated universal oriented fibration, and the conclusion follows.

For fibrations with unbased fibres, we develop orientation theory in terms of fibrewise suspension. That is, an orientation of $\xi: E \rightarrow B$ is defined to be an orientation of $\Sigma^{+} \xi$, where $\Sigma X$ is assumed to have an $R$-fundamental class. Define $\mathcal{E}(X ; R)(B)$ to be the resulting set of equivalence classes of oriented fibrations over $B$. 
COROllaRY 2.6. There is a natural bijection

$$
S \mathcal{E} X(B) \cong \mathcal{E}(X ; R)(B)
$$

provided that $\Sigma X$ has an $R$-fundamental class and that $\Sigma_{*}^{+}: \pi_{0} G X \rightarrow \pi_{0} F \Sigma^{+} X \cong$ $\pi_{0} F \Sigma X$ is an isomorphism.

Proof. With $Y$ defined for $\Sigma^{+} X$ as in the proof of the theorem, [14, 11.3] implies that $B(Y, G X, *)$ classifies $\mathcal{E}(X ; R)$. Clearly $B(Y, G X, *)$ is weakly equivalent to $B(F R, G X, *)$, and the hypothesis on $\pi_{0}$ ensures that the natural map

$$
B S G X \rightarrow B(F R, G X, *)
$$

is a weak equivalence. The conclusion follows.

3. The behavior of $B H X$ with respect to changes of $X$. We need a preliminary definition in order to state our main technical result, which gives $B H X$ a limited form of functoriality in $X$.

Definition 3.1. Let $X$ and $Y$ be nondegenerately based spaces in $W$. For $H=G, F$, etc. and for a map $f: X \rightarrow Y$, define $H(X, Y ; f)$ to be the space of maps $d: X \rightarrow Y$ such that $d$ is homotopic to $b f a$ for some maps $b \in H Y$ and $a \in H X$. Say that $f$ is a weak $H$-equivalence if composition by $f$,

$$
f^{*}: H Y \rightarrow H(X, Y ; f)
$$

is a weak homotopy equivalence.

The following result shows that "homotopical conjugation" $H X \rightarrow H Y$ is induced by a map $B H X \rightarrow B H Y$ whenever the former is defined. Let $\zeta: H X \rightarrow$ $\Omega B H X$ be the natural weak equivalence $[14,8.7]$.

Theorem 3.2. (i) $A$ weak $H$-equivalence $f: X \rightarrow Y$ determines a canonical map $f_{\#}$ : $B H X \rightarrow B H Y$ such that the following diagram commutes.

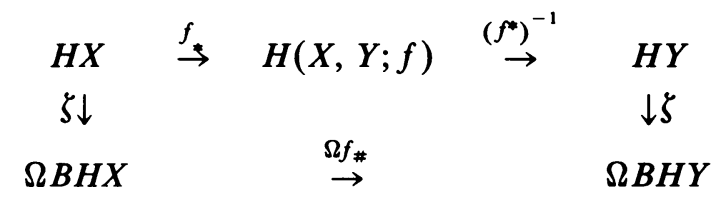

(ii) If $f$ is also a weak $H^{\prime}$-equivalence, $H X \subset H^{\prime} X$, then the following diagram commutes.

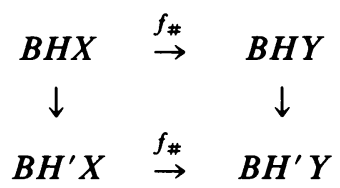

(iii) If $f: X \rightarrow Y, g: Y \rightarrow Z$, and $g f: X \rightarrow Z$ are weak $H$-equivalences, then the following diagram commutes.

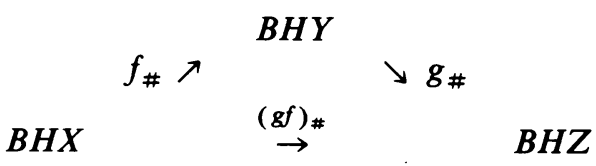


(iv) If $f: X \rightarrow Y, f^{\prime}: X^{\prime} \rightarrow Y^{\prime}, f * 1: X * Y^{\prime} \rightarrow Y * Y^{\prime}, 1 * f^{\prime}: Y * X^{\prime} \rightarrow Y * Y^{\prime}$, and $f * f^{\prime}:$ $X * X^{\prime} \rightarrow Y * Y^{\prime}$ are all weak G-equivalences, then the following diagram commutes, and similarly with $G$ replaced by $S G$ and with $G$ replaced by $F$ or $S F$ and join replaced by smash product.

$$
\begin{array}{cccc}
B G X \times B G X^{\prime} & \cong B\left(G X \times G X^{\prime}\right) & \stackrel{B(*)}{\rightarrow} & B G\left(X * X^{\prime}\right) \\
f_{\#} \times f_{\#}^{\prime} \downarrow & & & \downarrow\left(f * f^{\prime}\right)_{\#} \\
B G Y \times B G Y^{\prime} & \cong B\left(G Y \times G Y^{\prime}\right) & \stackrel{B(*)}{\rightarrow} & B G\left(Y * Y^{\prime}\right)
\end{array}
$$

Actually, the diagram of (i) will require a bit of interpretation to keep track of basepoints (as well as formal inverses, which are used implicitly in all parts of the theorem).

The rest of this section is devoted to the proof, which is based on the general categorical constructions introduced in $[14, \S 12]$. We refer the reader to that source for the construction of the two-sided bar construction $B(\mathcal{Y}, \mathcal{G}, \mathcal{X})$ determined by a topological category $\mathcal{G}$ with object space $\mathcal{O}$, a right $\mathcal{O}$-graph $\mathcal{Y}$ over $\mathcal{G}$, and a left $\mathcal{O}$-graph $\mathcal{X}$ over $\mathcal{G}$. We use these notions to circumvent the basic difficulty, which is simply that $f$ fails to induce a morphism of monoids $H X \rightarrow H Y$.

Let $\theta$ be the discrete space with two points $x$ and $y$. Let $\mathcal{G}=\mathcal{G}(f)$ be the topological category with object space $\theta$ and morphism spaces

$$
\mathcal{G}(x, x)=H X, \quad \mathcal{G}(y, y)=H Y, \quad \text { and } \quad \mathcal{G}(x, y)=H(X, Y ; f),
$$

with $\mathcal{G}(y, x)$ empty. This is a well-defined category under composition of maps. Let $\mathscr{F}=\mathscr{F}(f)$ be the right $\mathcal{\theta}$-graph over $\mathcal{G}$ the space of which is

$$
\mathcal{G}(x, y) \amalg \mathcal{G}(y, y)
$$

with the evident source map to $\mathcal{O}$ and with the right action of $\mathcal{G}$ being given by composition of maps. Regard $H X$ and $H Y$ as categories with unique objects $x$ and $y$, respectively, and let $i: H X \rightarrow \mathcal{G}$ and $j: H Y \rightarrow \mathcal{G}$ be the evident inclusions of categories. Let $j$ also denote the inclusion of $H Y$ in $\mathscr{F}$ and observe that composition with $f$ induces $f_{*}: H X \rightarrow \mathcal{F}$. We have the following commutative diagram of spaces and maps.

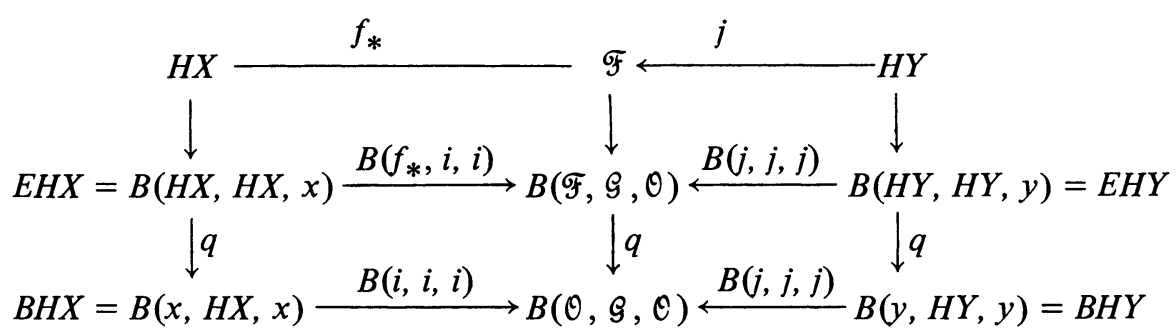

Here $B(\mathcal{O}, \mathcal{G}, \mathcal{O})=B \mathcal{G}$ is just the ordinary classifying space of $\mathcal{G}$.

On general nonsense grounds [13, 9.8 and 11.10], $E H X$ and $E H Y$ are contractible and the natural map (induced by the target function of the left hand variable G)

$$
\varepsilon: B(\mathcal{G}, \mathcal{G}, \mathcal{\theta}) \rightarrow \mathcal{O}
$$


is a homotopy equivalence. The component of $x$ in $B(\mathcal{G}, \mathcal{G}, \theta)$ is precisely $E H X$ embedded via $B(i, i, i)$. The component of $y$ is precisely $B(\mathscr{F}, \mathcal{G}, \mathcal{O})$, hence this space is also contractible.

Since $\pi_{0} H X$ and $\pi_{0} H Y$ are groups, the left and right maps $q$ of (A) are quasifibrations with fibres $H X$ and $H Y$. The middle map $q$ is also a quasifibration, by inspection of the proof of $[14,7.6]$. The point is that, for all morphisms $k$ of $\mathcal{G}$, the right translations by $k$ on the components of $\mathscr{F}$ are weak equivalences, this being obvious if $k \in H X$ or $k \in H Y$ and being true by hypothesis if $k \in H(X, Y$; f).

The comparison of quasifibrations displayed in (A) shows that $B j=B(j, j, j)$ is a weak equivalence and allows us to define $f_{\#}: B H X \rightarrow B H Y$ to be the formal composite given by the bottom row, namely $(B j)^{-1} B i$. To prove (i), consider the following expanded (and more accurate) version of the relevant diagram.

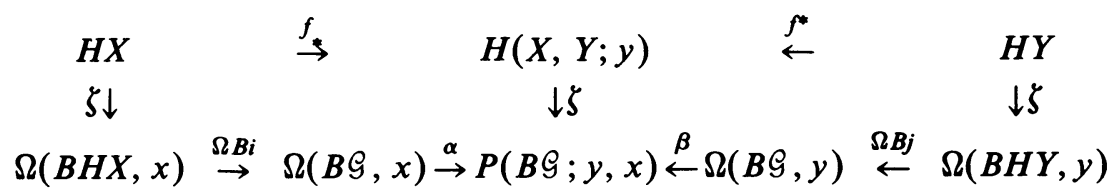

Here $x$ and $y$ are thought of as points of $B \mathcal{G}$, and the middle bottom space consists of paths in $B \mathcal{G}$ which start at $y$ and end at $x$. The maps $\zeta$ are all specified by

$$
\zeta(d)(t)=|[d],(t, 1-t)|, \quad(t, 1-t) \in \Delta_{1} .
$$

The maps $\alpha$ and $\beta$ are homotopy equivalences obtained by adding the path $\zeta(f)$ to loops in $B \mathcal{G}$ at the basepoints $x$ and $y$. By use of the maps $H X \times \Delta_{2} \rightarrow B \mathcal{G}$ and $H Y \times \Delta_{2} \rightarrow B \mathcal{G}$ specified by $(a, u) \rightarrow|[f \mid a], u|$ and $(b, u) \rightarrow|[b \mid f], u|$, one easily checks that the diagram homotopy commutes. This proves (i), or rather its adjusted statement necessary to account for the fact that $f_{\#}$ is not a formal composite of based maps.

Part (ii) is immediate by comparison of the diagrams (A) for $H$ and for $H^{\prime}$.

To prove the functoriality statement (iii), it suffices to construct a category $\overline{\mathcal{G}}$ and a commutative diagram of categories and functors

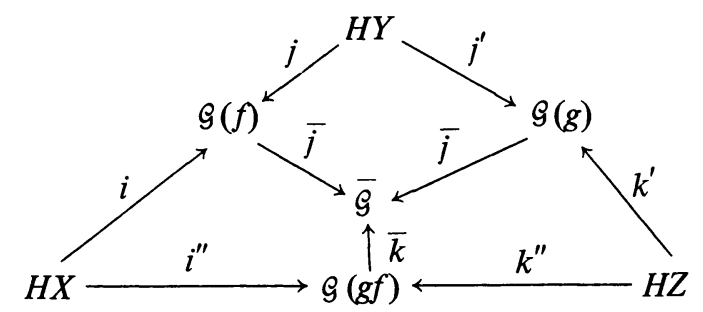

in which the outer arrows are the evident inclusions and the arrow $\bar{k}$ (as well as $j$, $k^{\prime}$, and $k^{\prime \prime}$ ) induces a weak equivalence on passage to classifying spaces. Thus let $\overline{\mathcal{G}}$ have object space $\overline{\mathcal{O}}=\{x, y, z\}$ and morphism space generated under composition by the union of the morphism spaces of $\mathcal{G}(f), \mathcal{G}(g)$, and $\mathcal{G}(g f)$. Note for this that 


$$
\begin{aligned}
\mathcal{G}(g f)(x, z) & =H(X, Z ; g f) \\
& =\{e: X \rightarrow Z \mid e \simeq c g f a \text { for some } c \in H Z \text { and } a \in H X\} \\
& =\{e: X \rightarrow Z \mid e \simeq c g b f a \text { for some } c \in H Z, b \in H Y, \text { and } a \in H X\}
\end{aligned}
$$

is to be taken as $\overline{\mathcal{G}}(x, z)$. The last equality holds since the fact that $g^{*}: H Z \rightarrow$ $H(Y, Z ; g)$ is a weak equivalence and therefore induces a bijection on components implies that if $b \in H Y$ then $g b \simeq c g$ for some $c \in H Z$. Let $\bar{i}, \bar{j}$ and $\bar{k}$ in (B) be the evident inclusions; then (B) certainly commutes. Let $\overline{\mathcal{F}}$ be the right $\mathcal{\theta}$-graph over $\overline{\mathcal{G}}$ with underlying space

$$
\overline{\mathcal{G}}(x, z) \amalg \overline{\mathcal{G}}(y, z) \amalg \overline{\mathcal{G}}(z, z) .
$$

Again, we have a homotopy equivalence

$$
\varepsilon: B(\overline{\mathcal{G}}, \overline{\mathcal{G}}, \overline{\mathcal{O}}) \rightarrow \mathcal{O},
$$

hence the component $B(\overline{\mathscr{F}}, \overline{\mathcal{G}}, \overline{\mathcal{O}})$ over $z$ is contractible. Moreover, the natural map

$$
q: B(\overline{\mathscr{F}}, \overline{\mathcal{G}}, \overline{\mathcal{O}}) \rightarrow B \overline{\mathcal{G}}
$$

is a quasifibration. Again, the point is that all right translations of components of $\bar{F}$ by morphisms of $\overline{\mathcal{G}}$ are weak equivalences, and, the key to the verification of this is just the commutative diagram

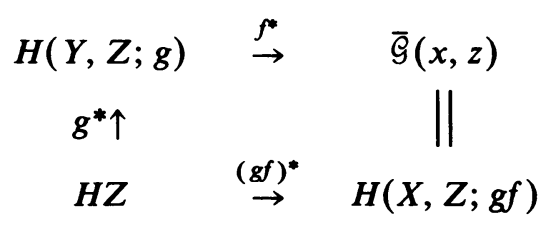

Thus $B \bar{k}$ is a weak equivalence by a comparison of quasifibrations.

To prove the join statement (iv), it suffices to construct a category $\tilde{\mathcal{G}}$ and a commutative diagram of categories and functors

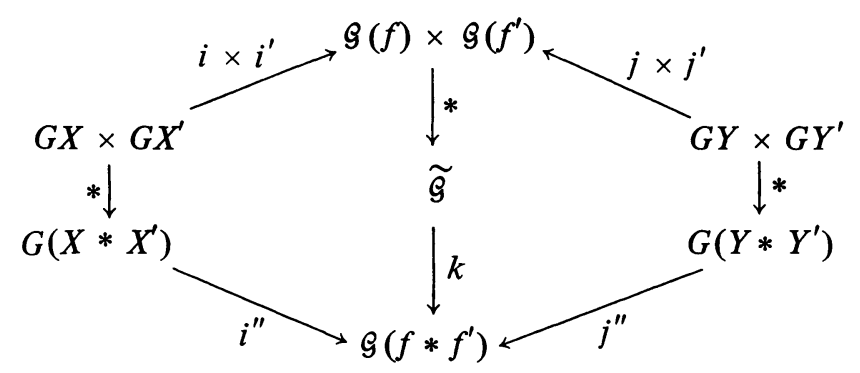

in which the $i$ 's and $j$ 's are the evident inclusions and the arrow $k$ (as well as the $j$ 's) induces a weak equivalence on passage to classifying spaces. Thus let $\tilde{\mathcal{G}}$ have (discrete) object space

$$
\tilde{\mathcal{\theta}}=\left\{x * x^{\prime}, x * y^{\prime}, y * x^{\prime}, y * y^{\prime}\right\}
$$


and morphism spaces

$$
\begin{array}{rlrl}
\tilde{\mathcal{G}}\left(x * x^{\prime}, x * x^{\prime}\right) & =G\left(X * X^{\prime}\right), & \tilde{\mathcal{S}}\left(y * y^{\prime}, y * y^{\prime}\right) & =G\left(Y * Y^{\prime}\right), \\
\tilde{\mathcal{G}}\left(x * x^{\prime}, x * y^{\prime}\right) & =G\left(X * X^{\prime}, X * Y^{\prime} ; 1 * f^{\prime}\right), & \tilde{\mathcal{S}}\left(x * x^{\prime}, y * x^{\prime}\right) & =G\left(X * X^{\prime}, Y * X^{\prime} ; f * 1\right), \\
\tilde{\mathcal{G}}\left(x * y^{\prime}, y * y^{\prime}\right) & =G\left(X * Y^{\prime}, Y * Y^{\prime} ; f * 1\right), & \tilde{\mathcal{G}}\left(y * x^{\prime}, y * y^{\prime}\right) & =G\left(Y * X^{\prime}, Y * Y^{\prime} ; 1 * f^{\prime}\right), \\
\tilde{\mathcal{G}}\left(x * x^{\prime}, y * y^{\prime}\right)=G\left(X * X^{\prime}, Y * Y^{\prime} ; f * f^{\prime}\right) ;
\end{array}
$$

the remaining morphism spaces are empty. Closure under composition results from the facts that if $c \in H\left(X * Y^{\prime}\right)$, then $(f * 1) c \simeq b(f * 1)$ for some $b \in G\left(X * X^{\prime}\right)$ and symmetrically for $d \in H\left(Y * X^{\prime}\right)$. Join of maps specifies $*: \mathcal{G}(f) \times \mathcal{G}\left(f^{\prime}\right) \rightarrow \tilde{\mathcal{G}}$, and $k: \tilde{\mathcal{G}}\left(f * f^{\prime}\right) \rightarrow \tilde{\mathcal{G}}$ is the evident inclusion; certainly (C) commutes. Let $\tilde{\mathscr{F}}$ be the right $\tilde{\mathcal{O}}$-graph over $\tilde{\mathcal{G}}$ with underlying space

$$
\tilde{\mathcal{G}}\left(x * x^{\prime}, y * y^{\prime}\right) \amalg \tilde{\mathcal{G}}\left(x * y^{\prime}, y * y^{\prime}\right) \amalg \tilde{\mathcal{G}}\left(y * x^{\prime}, y * y^{\prime}\right) \amalg \tilde{\mathcal{G}}\left(y * y^{\prime}, y * y^{\prime}\right) \text {. }
$$

Just as in our previous arguments, $B(\tilde{\mathscr{F}}, \tilde{\mathcal{G}}, \tilde{\mathcal{O}})$ is contractible and the natural map $q: B(\tilde{\mathscr{F}}, \tilde{\mathcal{G}}, \tilde{\mathcal{O}}) \rightarrow B \tilde{\mathcal{S}}$ is a quasifibration. For the latter, the key to verifying that the relevant right translations are weak equivalences is just the commutative diagram

$$
\begin{aligned}
& G\left(X * Y^{\prime}, Y * Y^{\prime} ; f * 1\right) \stackrel{\left(1 * f^{\prime}\right)^{*}}{\rightarrow} \tilde{\mathcal{G}}\left(x * x^{\prime}, y * y^{\prime}\right) \stackrel{(f * 1)^{*}}{\leftarrow} G\left(Y * X^{\prime}, Y * Y^{\prime} ; 1 * f^{\prime}\right) \\
& (f * 1) * \uparrow \quad \uparrow\left(1 * f^{\prime}\right)^{*} \\
& G\left(Y * Y^{\prime}\right) \quad \stackrel{\left(f * f^{\prime}\right)^{*}}{\rightarrow} G\left(X * X^{\prime}, Y * Y^{\prime} ; f * f^{\prime}\right) \stackrel{\left(f * f^{\prime}\right)^{*}}{\leftarrow} G\left(Y * Y^{\prime}\right)
\end{aligned}
$$

Thus $B k$ is a weak equivalence by a comparison of quasifibrations.

4. Fibrewise localization and completion. Before specializing the theory above, we point out one way not to proceed (since incorrect assertions on this score appear several places in the literature). There exist constructions of localizations and completions which are functorial on the space level, before passage to homotopy. Such constructions yield homomorphisms of monoids

$$
F X \rightarrow F X_{T} \text { and } F X \rightarrow F \hat{X}_{T} .
$$

That is, a based homotopy equivalence $X \rightarrow X$ localizes to a based homotopy equivalence $X_{T} \rightarrow X_{T}$, and functoriality implies that composition is preserved. However, we cannot pass to classifying spaces because there are no known continuous localization and completion functors. That is, there is no reason to expect the homomorphisms above to be continuous. No constructions which pass through the world of simplicial sets can possibly be continuous, and direct inspection shows that the other known localization functors also fail to be continuous.

We fall back on the theory already developed. Let $X$ be a (connected) nilpotent nondegenerately based space in $थ$. Let $\lambda: X \rightarrow X_{T}$ and $\gamma: X \rightarrow \hat{X}_{T}$ be a localization and a completion of $X$ at a set of primes $T$, where $X_{T}$ and $\hat{X}_{T}$ are also nondegenerately based spaces in $\Psi$, and recall that we may take $\gamma$ to be $\gamma \lambda$, where $\gamma: X_{T} \rightarrow \hat{X}_{T}$ is a completion of $X_{T}$ at $T$. 
THEOREM 4.1 (FIBREWISE LOCALIZATION AND COMPLETION).

(i) For $H=F, G, S F$, and $S G$, there is a commutative diagram

$$
\begin{array}{lcccc} 
& & B H X_{T} & & \\
& \lambda_{\#} \nearrow & & \searrow \gamma_{\#} & \\
& \stackrel{\gamma_{\#}}{\rightarrow} & & B H \hat{X}_{T}
\end{array}
$$

These diagrams are compatible with the inclusions $H \subset H^{\prime}$ and the diagrams for $S F$ and $S G$ are homotopy universal covers of the diagrams for $F$ and $G$.

(ii) For $H=F$, the induced diagram of fundamental groups of (i) is

$$
\begin{array}{lcccc} 
& & \pi_{0} F X_{T} & & \\
& \nearrow & & \searrow & \\
\pi_{0} F X & & \rightarrow & & \pi_{0} F \hat{X}_{T}
\end{array}
$$

where the arrows are given by localization and completion of homotopy classes of based homotopy equivalences.

(iii) If $X$ is a finite $\mathrm{CW}$-complex, or a finite $S$-local $\mathrm{CW}$-complex for any set of primes $S \supseteq T$, then, in the diagram of (i) for $H=S F$ or $S G, \lambda_{\#}$ is a localization at $T$ and the $\gamma_{\#}$ are completions at $T$.

(iv) The following diagram commutes, and similarly with $G$ replaced by $S G$ and with $G$ replaced by $F$ or $S F$ and joins replaced by smash products.

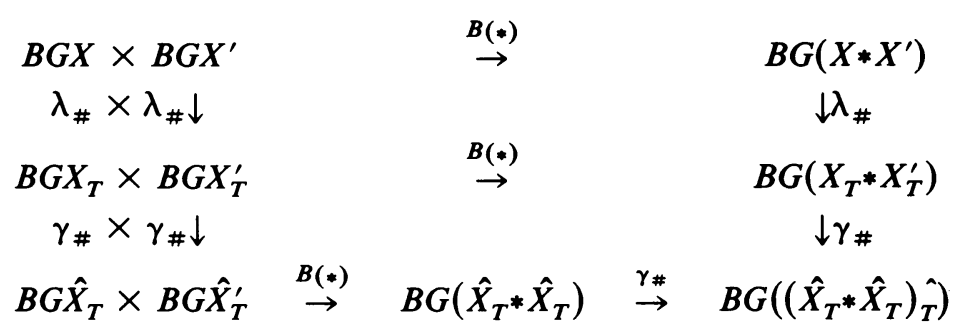

Note in (ii) that $\pi_{0} F X=\pi_{0} G X$ for all simply connected $X$; in general, $\pi_{0} G X$ is the orbit set of $\pi_{0} F X$ under the action of $\pi_{1} X$ in the fibration $F X \rightarrow G X \rightarrow X$.

The theorem simulaneously constructs fibrewise localizations and completions by direct appeal to the classification theorem and interprets their behavior on the level of classifying spaces. If $\xi: E \rightarrow B$ is a fibration with fibre $X$ classified by $\alpha$ : $B \rightarrow B H X$, then the fibrewise localization of $\xi$ is the fibration $\xi_{T}^{f}: E_{T}^{f} \rightarrow B$ classified by $\lambda_{\#} \alpha: B \rightarrow B H X_{T}$. By tracing through the constructions on the space level (all relevant spaces coming with canonical universal fibrations over them) and exploiting the arguments used to prove Lemma 1.1, one finds easily that there is a natural fibrewise map $\lambda_{\#}: E \rightarrow E_{T}^{f}$ which localizes fibres. Moreover, these constructions on fibrations commute with fiberwise joins and smash products. The rest of the theorem can be interpreted similarly on the fibration level.

The rest of this section is devoted to the proof. I owe the following key result to Pete Bousfield. Recall that $F(X, Z)$ denotes the space of based maps $X \rightarrow Z$. 
Proposition 4.2. If $f: X \rightarrow Y$ induces an isomorphism on $T$-local cohomology (resp., on $\bmod p$ cohomology for all primes $p \in T$ ) and $Z$ is $T$-local (resp., T-complete), then

$$
f^{*}: F(Y, Z) \rightarrow F(X, Z)
$$

is a weak homotopy equivalence.

Proof. We may assume without loss of generality that $f$ is a cofibration. It suffices to show that if $i: K \rightarrow L$ is a cofibration in $W$ and $f^{*} h=g i$ in the following diagram, then there is a map $j$ which makes the diagram commute:

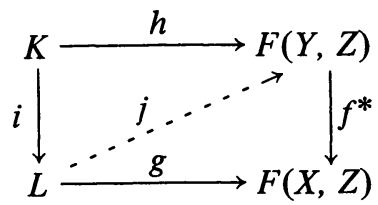

Passing to adjoints, it suffices to show that any map $k$ in the following diagram extends to a map $\tilde{k}$ :

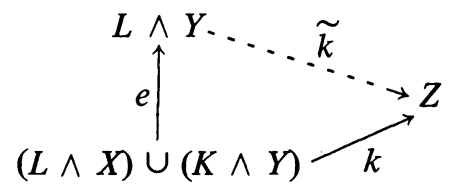

Here the cofibration $e=(1 \wedge f) \cup(i \wedge 1)$ inherits the cohomological property assumed on $f$. Indeed,

$$
1 \wedge f: K \wedge X \rightarrow K \wedge Y \text { and } 1 \wedge f: L \wedge X \rightarrow L \wedge Y
$$

are $T$-local (resp., $\bmod p$ for $p \in T$ ) cohomology isomorphisms since $f$ is, and the corresponding assertion for $e$ follows easily by use of the relative Mayer-Vietoris sequence. Now the existence of $\bar{k}$ such that $\bar{k} e \simeq k$ is immediate from the standard characterization of $T$-local or $T$-complete spaces [3, V.3.3 and VI.5.4]; [16]; [21]. Since $e$ is a cofibration, so that its mapping cylinder is a retract of $(L \wedge Y) \times I$, the existence of $\tilde{k}$ as required follows.

Recall Definition 3.1. If $f: X \rightarrow Y$ is a localization or completion, then any based map $g: X \rightarrow Y$ is homotopic to a composite $\tilde{g} f$, by the defining universal property, and $\tilde{g}$ is an equivalence if $g$ is a $T$-local or $\bmod p$ for $p \in T$ cohomology isomorphism. A moment's reflection (to the effect that any free equivalence is freely homotopic to a based equivalence) shows that, for any of our $H, H(X, Y ; f)$ is a union of some of the components of $F(X, Y)$ or $G(X, Y)$. Either noting that the proof above works equally well in the free case or using the comparison of fibrations

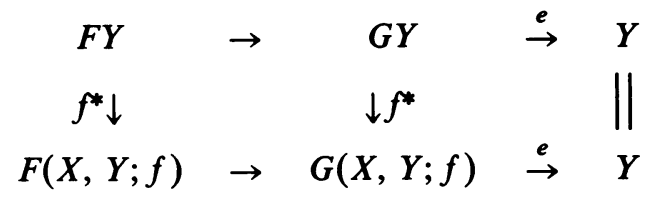


we conclude from the proposition that any localization or completion is a weak $H$-equivalence for any of our $H$. Thus the construction of the maps $\lambda_{\#}$ and $\gamma_{\#}$ of Theorem 4.1 is immediate from Theorem 3.2(i).

Theorem 4.1(i) follows from Theorem 3.2 and Proposition 2.1 and Theorem 4.1(ii) is immediate from the diagram in Theorem 3.2(i). In view of the following result, Theorem 4.1(iii) also follows directly from the cited diagram by the standard characterizations of localizations and completions [3, V.4.1 and V.6.1]; [10, II.3B]; [16]; [21].

Proposition 4.3. Let $X$ be a finite $\mathrm{CW}$-complex or a finite $S$-local $\mathrm{CW}$-complex for any set of primes $S \supseteq T$. If $f: X \rightarrow Y$ is a localization or completion at $T$, then so is

$$
f_{*}: S H X \rightarrow S H(X, Y ; f), \quad H=F \text { or } G
$$

Proof. Observe that $\operatorname{SF}(X, Y ; f)$ is just the component of $f$ in $F(X, Y)$, and similarly for $G$. For ordinary $C W$-complexes, the conclusion for $F$ is a standard result [3, V §5 and VI §7]; [10, p. 77]; [16]. The conclusion for $G$ follows by the comparison of fibrations

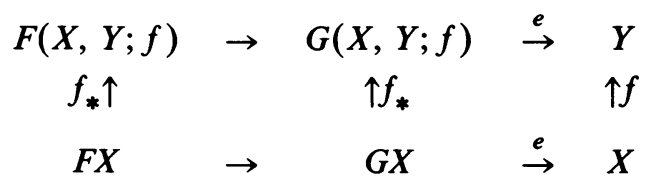

The proof for $S$-local CW-complexes, a thorough study of which will appear in [16], is exactly the same.

It remains to prove Theorem 4.1(iv). Since localization commutes with joins and smash products, the upper square commutes by Theorem 3.2(iv). For the bottom square, in which we may as well replace $X_{T}$ by $X$, we go back to the drawing boards.

To simplify and mesh notations, let $f: X \rightarrow Y$ and $f^{\prime}: X^{\prime} \rightarrow Y^{\prime}$ be completions at $T$. We may still construct diagram (C) of the previous section, but the functors $j^{\prime \prime}$ and $k$ there no longer induce weak equivalences on passage to classifying spaces. Consider a homotopy commutative diagram

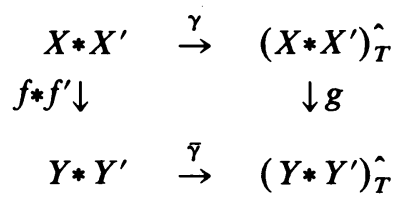

in which $\gamma$ and $\bar{\gamma}$ are completions at $T$ and thus $g$ is $\left(f * f^{\prime}\right)_{T}$. Then $g$ is a $\bmod p$ cohomology isomorphism for $p \in T$ and is thus an equivalence (this being the standard best that one can say in general about the behavior of joins and smash products with respect to completions). We embed diagram (C) in the following larger diagram, in which the arrows labelled $\simeq$ induce weak equivalences on passage to classifying spaces. 


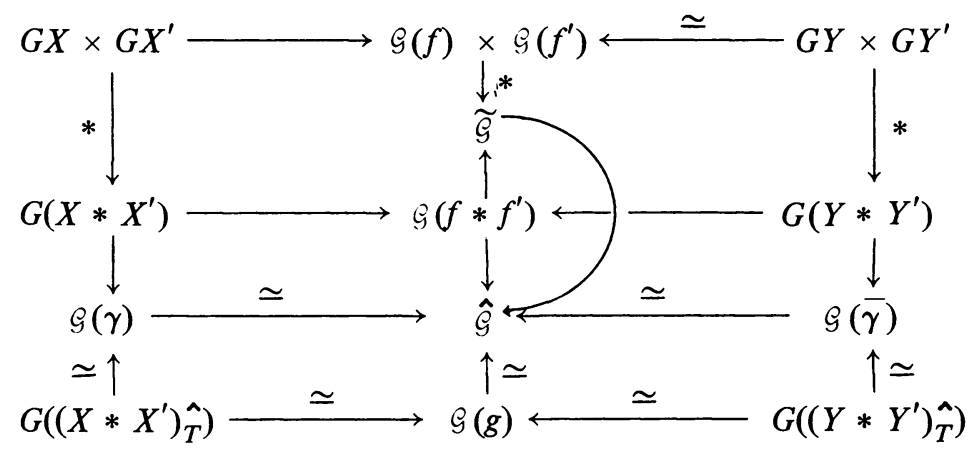

Here $\hat{\mathcal{S}}$ is the category generated by the five categories $\hat{\mathcal{G}}, \mathcal{G}(\gamma), \mathcal{G}\left(f * f^{\prime}\right), \mathcal{G}(\bar{\gamma})$, and $\mathcal{G}(g)$. That is, $\hat{\mathcal{G}}$ has (discrete) object space

$$
\hat{\mathcal{O}}=\left\{x * x^{\prime}, x * y^{\prime}, y * x^{\prime}, y * y^{\prime},\left(x * x^{\prime}\right)_{T},\left(y * y^{\prime}\right)_{T}\right\}
$$

with morphism spaces dictated by closure under composition and containment of the cited five categories. As in the arguments of the previous section, to prove that the three arrows marked $\simeq$ landing in $\hat{\mathcal{G}}$ induce weak equivalences on passage to classifying spaces, one uses comparisons of quasifibrations. That is, one forms the right $\hat{\mathcal{O}}$-graph $\hat{\mathscr{F}}$ over $\hat{\mathcal{G}}$ consisting of those morphisms with target $\left(y * y^{\prime}\right)_{T}$, observes that $B(\hat{\mathscr{F}}, \hat{\mathcal{G}}, \hat{\mathcal{O}})$ is contractible, and checks that

$$
q: B(\hat{\mathscr{F}}, \hat{\mathcal{G}}, \hat{\mathcal{O}}) \rightarrow B \hat{\mathcal{G}}
$$

is a quasifibration by using Proposition 4.2 to verify that the relevant right translations are weak equivalences. The diagram in Theorem 4.1(iv) follows on passage to classifying spaces since the resulting map $B G\left(X * X^{\prime}\right) \rightarrow B G\left(\left(Y * Y^{\prime}\right)_{T}\right)$, namely $g_{\#} \gamma_{\#}$, may be viewed as $\gamma_{\#}$ because $g \gamma$ is a completion (and completion is in any case only well defined up to equivalence of its target).

5. Spherical fibrations. The main applications so far have dealt with spherical fibrations, to which we now specialize. The real work has already been done, and we show here how it implies various of Sullivan's assertions in [20] and Dold's theorem $\bmod k$. We shall have several uses for the following elementary facts.

LEMMA 5.1. (i) If $Y$ is a T-local space, then

$$
\pi_{q} F\left(S_{T}^{n}, Y\right)=\pi_{n+q} Y
$$

(ii) If $Y$ is a $T$-complete space, then

$$
\pi_{q} F\left(\hat{S}_{T}^{n}, Y\right)=\pi_{n+q} Y .
$$

Proof. Clearly $\pi_{q} F(X, Y)$ is the set of homotopy classes of based maps $\Sigma^{q} X \rightarrow$ $Y$. Part (i) is clear from $\Sigma^{q} S_{T}^{n}=S_{T}^{n+q}$ and the universal property of localization. Part (ii) is clear from $\left(\Sigma^{q} \hat{S}_{T}^{n}\right)_{T}=\hat{S}_{T}^{n+q}$ and the universal property of completion.

Let $Z_{T}$ and $\hat{Z}_{T}=X_{p \in T} \hat{Z}_{(p)}$ denote the localization and completion of the integers at $T$. When $X=S^{n}$ for $n \geqslant 1$, the diagram of fundamental groups of Theorem 4.1(ii) applies to both $F X$ and $G X$ and, by the case $q=0$ of the lemma, 
reduces to the diagram of units

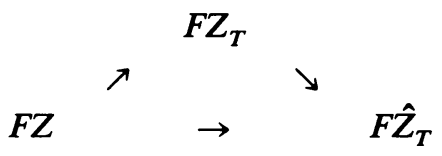

Of course, $F Z \cong Z_{2}, F Z_{T} \cong Z_{2} \oplus\left(\bigoplus_{p \in T} Z\right)$, and $F \hat{Z}_{T} \cong X_{p \in T} F \hat{Z}_{(p)}$, where $F \hat{Z}_{(2)}$ $\simeq Z_{2} \oplus \hat{Z}_{(2)}$ and $F \hat{Z}_{(p)} \cong Z_{p-1} \oplus \hat{Z}_{(p)}$ for $p>2$.

Clearly $\lambda: S^{n} \rightarrow S_{T}^{n}$ and $\gamma: S^{n} \rightarrow \hat{S}_{T}^{n}$ are $Z_{T}$ and $\hat{Z}_{T}$ fundamental classes in the sense of Definition 2.2. By Theorem 2.4, the homotopy universal covers $B S F\left(S_{T}^{n}\right)$ and $B S F\left(\hat{S}_{T}^{n}\right)$ of $B F\left(S_{T}^{n}\right)$ and $B F\left(\hat{S}_{T}^{n}\right)$ classify $Z_{T}$ and $\hat{Z}_{T}$ oriented fibrations with based fibres $S_{T}^{n}$ and $\hat{S}_{T}^{n}$. By Corollary 2.5 , the actions of $F Z_{T}$ and $F \hat{Z}_{T}$ by covering transformations correspond under this classification to their actions on the orientations of oriented fibrations.

Since $\Sigma S_{T}^{n}=S_{T}^{n+1}$, Corollary 2.5 implies that $B S G\left(S_{T}^{n}\right)$ classifies $Z_{T}$ oriented fibrations with unbased fibres $S_{T}^{n}$. Since we only have $\left(\Sigma \hat{S}_{T}^{n} \hat{T}_{T}^{n}=\hat{S}_{T}^{n+1}\right.$, we define an orientation of a fibration with unbased fibres $\hat{S}_{T}^{n}$ to be an orientation of the fibrewise completion at $T$ of its fibrewise suspension. Here, modulo the evident interpretation needed to account for formal inverses, we find by a diagram chase that the diagram

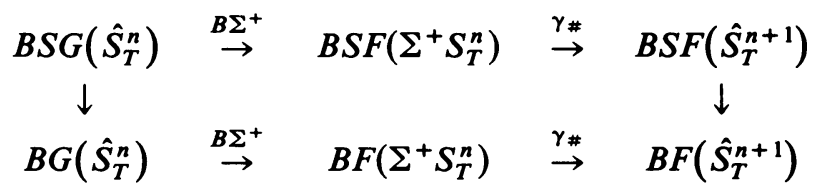

is a homotopy pullback; such a comparison of universal covers is necessarily a pullback in the homotopy category. Since an oriented fibration over $B$ is classified by a map $\alpha: B \rightarrow B G\left(\hat{S}_{T}^{n}\right)$ together with a lift of $\gamma_{\#} \circ B \Sigma^{+} \circ \alpha$ to $B S F\left(\hat{S}_{T}^{n+1}\right)$, it follows that $B S G\left(\hat{S}_{T}^{n}\right)$ classifies $\hat{Z}_{T}$ oriented fibrations with unbased fibres $\hat{S}_{T}^{n}$. Again, by comparisons of universal covers, the covering transformation actions by units on $B S G\left(S_{T}^{n}\right)$ and $B S G\left(\hat{S}_{T}^{n}\right)$ correspond to the actions by units on orientations.

We have already observed that (based) oriented $S_{T}^{n}$ and $\hat{S}_{T}^{n}$ fibrations have $Z_{T}$ and $\hat{Z}_{T}$ cohomology Thom isomorphisms, by the Serre spectral sequence.

Of course, the calculational power of the theory lies in the equivalences

$$
B S H\left(S_{T}^{n}\right) \simeq\left(B S H\left(S^{n}\right)\right)_{T} \text { and } B S H\left(\hat{S}_{T}^{n}\right) \simeq\left(B S H\left(S^{n}\right) \hat{T}_{T}\right.
$$

for $H=F$ and $G$ and the finiteness of the homotopy groups involved. Recall the standard notations

$$
G(n)=G\left(S^{n-1}\right), \quad S G(n)=S G\left(S^{n-1}\right), \quad F(n)=F\left(S^{n}\right), \quad S F(n)=S F\left(S^{n}\right) .
$$

The point is that $O(n)$ maps to $G(n)$ by restriction of orthogonal transformations to the unit sphere and maps to $F(n)$ by one-point compactification of maps, these inclusions being compatible with suspension $G(n) \rightarrow F(n)$ by virtue of an evident homeomorphism $\Sigma^{+} S^{n-1} \cong R^{n} \cup \infty$. Since $S F(n)$ is a component of $\Omega^{n} S^{n}$, $\pi_{q} S F(n)=\pi_{n+q} S^{n}$ for $q>0$. The fibrations $S^{n} \rightarrow B S F(n) \rightarrow B S G(n+1)$ imply that the groups $\pi_{q} B S F(n)$ and $\pi_{q} B S G(n)$ are all finite except $\pi_{n} B S F(n)$ and $\pi_{n} B S G(n)$ for even $n \geqslant 2$ and $\pi_{2 n-2} B S G(n)$ for odd $n \geqslant 3$, each of which has a 
summand $Z$. (For even $n$, one checks that $S^{n} \rightarrow B S F(n)$ maps $\pi_{n}$ monomorphically by considering the Euler class.)

COROLlaRY 5.2. The rationalizations of the $B S F(n)$ and $B S G(n)$ are

$$
\begin{aligned}
B S G(2 n)_{0} & \simeq B S F(2 n)_{0} \simeq K(Q, 2 n), \\
B S G(2 n+1)_{0} & \simeq K(Q, 4 n), \quad B S F(2 n+1)_{0} \simeq\{*\} .
\end{aligned}
$$

Since the rational cohomology of $K(Q, 2 n)$ is the polynomial algebra on one generator of degree $2 n$, it is clear from the Gysin sequence of $S^{2 n} \rightarrow B S F(2 n) \rightarrow$ $B S G(2 n+1)$ that a generator of $H^{4 n}(B S G(2 n+1) ; Q)$ can be chosen which pulls back to the square of the Euler class. Of course, these characteristic classes classify rational spherical fibrations.

Theorem 4.1(iv) gives basic commutation relations relating fibrewise joins and smash products to fibrewise localizations and completions. Fibrewise suspension carries $S_{T}^{n}$-fibrations to $S_{T}^{n+1}$-fibrations and fibrewise suspension followed by fibrewise completion carries $\hat{S}_{T}^{n}$-fibrations to $\hat{S}_{T}^{n+1}$-fibrations. This applies to both based and unbased fibres, and the results of $\$ \S 2$ and 4 prove all reasonable commutation relations among these operations and between them and fibrewise joins and smash products.

In particular, we can form the telescopes

$$
B H=\text { Tel } B H\left(S^{n}\right), \quad B H_{T}=\operatorname{Tel} B H\left(S_{T}^{n}\right), \quad B H_{T}^{\wedge}=\operatorname{Tel} B H\left(\hat{S}_{T}^{n}\right)
$$

for $H=F, S F, G$, and $S G$. These classify the evident stable equivalence classes of spherical fibrations over finite-dimensional $\mathrm{CW}$-complexes. By the standard inverse limit arguments, the relevant diagrams commuting by the results cited above and the relevant $\lim ^{1}$ terms vanishing by the finiteness of the homotopy groups involved, fibrewise join and smash product (followed by fibrewise completion in the case of $B H_{T}$ ) give all of these spaces structures of homotopy associative and homotopy commutative $H$-space, these structures being compatible with all maps in sight.

Proposition 5.3. (i) $B F\left(S^{1}\right), B F\left(S_{T}^{1}\right)$, and $B F\left(\hat{S}_{T}^{1}\right)$ are $K(\pi, 1)$ 's, the respective groups $\pi$ being $F Z, F Z_{T}$, and $F \hat{Z}_{T}$.

(ii) The following composites are equivalences

$$
\begin{array}{lllll}
B F\left(S^{1}\right) \times B S F & \stackrel{\iota \times \pi}{\rightarrow} & B F \times B F & \stackrel{\phi}{\rightarrow} & B F, \\
B F\left(S_{T}^{1}\right) \times B S F_{T} & \stackrel{\iota \times \pi}{\rightarrow} & B F_{T} \times B F_{T} & \stackrel{\phi}{\rightarrow} & B F_{T}, \\
B F\left(\hat{S}_{T}^{1}\right) \times B S F_{T} & \stackrel{\iota \times \pi}{\rightarrow} & B F_{T} \times B F_{T} & \stackrel{\phi}{\rightarrow} & B F_{T},
\end{array}
$$

where the $\iota, \pi$, and $\phi$ are the natural inclusions, homotopy universal covers, and multiplications.

(iii) $B S F_{T} \simeq \times_{p \in T} B S F_{p}$ and $\gamma_{\#}: B S F_{T} \rightarrow B S F_{T}$ is an equivalence.

(iv) The following maps are equivalences

$$
B F \rightarrow B G, \quad B F_{T} \rightarrow B G_{T}, \quad B F_{T}^{n} \rightarrow B G_{T}^{\wedge},
$$

and similarly with $F$ and $G$ replaced by $S F$ and $S G$. 
Proof. Part (i) is immediate from Lemma 5.1. Part (ii) holds because $\phi(\iota \times \pi)$ obviously induces an isomorphism on homotopy groups (since $\phi$ induces addition). $S F$ is a component of $Q S^{0}=\lim _{\rightarrow} \Omega^{n} S^{n}$, hence $\pi_{q} S F$ is the $q$ th stable homotopy group of spheres and thus finite. This implies part (iii), and part (iv) follows from Proposition 2.1.

Thus the localizations $B S F_{p}$ determine all remaining stable classifying spaces in sight. In particular, the following consequence is often useful.

COROLlaRY 5.4. Fibrewise localization or completion at $T$ of oriented stable spherical fibrations over a finite $\mathrm{CW}$-complex $B$ is represented by the projection of the finite Abelian group $[B, B S F]$ on the product over $p \in T$ of its p-primary components $\left[B, B S F_{p}\right]$.

Of course, if $\operatorname{dim} B<n$, then $[B, B S F(n)] \cong[B, B S F]$ by the Freudenthal suspension and Whitehead theorems.

Versions of the following consequence have been noticed by several authors, notably Kahn [12, §2].

Proposition 5.5. Let $\zeta: D \rightarrow B$ and $\xi: E \rightarrow B$ be oriented fibrations with (based) fibre $S^{n}$ over a finite $\mathrm{CW}$-complex $B$. Consider the following statements.

(i) There is a fibrewise map $f: D \rightarrow E$ such that the degree of $f$ on each fibre is a unit $k \in Z_{T}$.

(ii) There is a fibrewise map $f_{T}: D_{T}^{f} \rightarrow E_{T}^{f}$ which restricts to a homotopy equivalence on each fibre.

(iii) $\zeta_{T}$ and $\xi_{T}$ are fibre homotopy equivalent.

(iv) There is a positive integral unit $m \in Z_{T}$ such that the $m$-fold Whitney sums $m \zeta$ and $m \xi$ are fibre homotopy equivalent.

The following implications hold:

$$
\text { (i) } \Rightarrow \text { (ii) } \Leftrightarrow \text { (iii) } \Rightarrow \text { (iv). }
$$

If $\operatorname{dim} B<n$, then all four assertions are equivalent.

Proof. We use Lemma 1.1 to ensure that all fibres and total spaces are in $W$. We use orientations of $\zeta$ and $\xi$ to fix orientations of fibres and so make sense of (i). Now (i) $\Rightarrow$ (ii) is immediate from the functoriality of fibrewise localizations and (ii) $\Leftrightarrow$ (iii) is a special case of Dold's theorem [5, 6.3]; $[14,2.6]$. For (iii) $\Rightarrow$ (iv), we may take $m=s t$ where $s$ is sufficiently large that $s \zeta$ and $s \xi$ are in the stable range and $t$ is the order of the torsion prime to $T$ in $[B, B S F]$; since $s\left(\zeta_{T}-\xi_{T}\right)=0$, the corollary above clearly gives $m(\zeta-\xi)=0$. The implication (iv) $\Rightarrow$ (iii) in the stable range is also immediate from the corollary. A nice proof that (ii) $\Rightarrow$ (i) when $\xi$ is trivial is given by Kahn [12, p. 210]. It follows for general $\xi$ that if $\operatorname{dim} B<n$ and (iv) holds, then there is a fibrewise map $\zeta \oplus \varepsilon_{n} \rightarrow \xi \oplus \varepsilon_{n}$ with degree on each fibre a unit $k \in Z_{T}$, where $\varepsilon_{n}$ is the trivial $S^{n}$-fibration. Indeed, we need only apply the implication (iv) $\Rightarrow$ (i) to $\varepsilon_{n}$ and $\zeta-\xi$ (which may be taken to be an $S^{n}$-fibration) and then add back $\xi$. Finally, to complete the proof that (ii) implies (i) in the stable range, we must show how to cancel $\varepsilon_{n}$ and so obtain $\zeta \rightarrow \xi$. That is, we must show that fibrewise suspension gives an isomorphism from the set of homotopy classes of 
fibrewise maps $\zeta \rightarrow \xi$ to that of fibrewise maps $\zeta \oplus \varepsilon_{n} \rightarrow \xi \oplus \varepsilon_{n}$. This was implicitly assumed by Kahn $[12, \S 2]$. As he explained to me, it can be proven as follows. If $\zeta$ and $\xi$ are fibre bundles, then the constructions in James [11] reduce the question to one about sections of certain fibrations, and the result follows by standard obstruction theory. Use of Fadell's theorem [7] then reduces the general case to the bundle case.

The arguments work equally well with $F$ replaced by $G$. The implication (i) $\Rightarrow$ (iv) is Adams' result $[1,1.1]$, namely Dold's theorem mod $k$. Here, taking $T$ to be the set of primes not dividing $k$ in (i), we clearly can take $m$ to be a power of $k$ in (iv).

We have been resolutely elementary in this paper. The reader interested in the deeper infinite loop analysis of the spaces $B S F_{p}$ is referred to [15].

\section{BIBLIOGRAPHY}

1. J. F. Adams, On the groups $J(X)-I$, Topology 2 (1963), 181-195.

2. G. Allaud, On the classification of fibre spaces, Math. Z. 92 (1966), 110-125.

3. A. K. Bousfield and D. M. Kan, Homotopy limits, completions and localizations, Lecture Notes in Math., vol. 304, Springer-Verlag, Berlin and New York, 1972.

4. R. Bruner, G. Lewis, J. P. May, J. McClure and M. Steinberger, $H_{\infty}$ ring spaces and their applications, Lecture Notes in Math., Springer-Verlag, Berlin and New York, in preparation.

5. A. Dold, Partitions of unity in the theory of fibrations, Ann. of Math. (2) 78 (1963), 223-225.

6. __ Halbexact Homotopiefunktoren, Lecture Notes in Math., vol. 12, Springer-Verlag, Berlin and New York, 1966.

7. E. Fadell, The equivalence of fibre spaces and bundles, Bull. Amer. Math. Soc. 66 (1960), 50-53.

8. H. Glover and G. Mislin, Immersion in the metastable range and 2-localization, Proc. Amer. Math. Soc. 43 (1974), 443-448.

9. D. H. Gottlieb, The total space of universal fibrations, Pacific J. Math. 46 (1973), 415-417.

10. P. Hilton, G. Mislin and J. Roitberg, Localization of nilpotent groups and spaces, Mathematics Studies, vol. 15, North-Holland, Amsterdam, 1975.

11. I. James, The space of bundle maps, Topology 2 (1963), 45-59.

12. P. J. Kahn, Mixing homotopy types of manifolds, Topology 14 (1975), 203-216.

13. J. P. May, The geometry of iterated loop spaces, Lecture Notes in Math., vol. 271, Springer-Verlag, Berlin and New York, 1972.

14. __ Classifying spaces and fibrations, Mem. Amer. Math. Soc., No. 155, 1975.

15. (with contributions by F. Quinn, N. Ray and J. Tornehave), $E_{\infty}$ ring spaces and $E_{\infty}$ ring spectra, Lecture Notes in Math., vol. 577, Springer-Verlag, Berlin and New York, 1977.

16. The homotopical foundations of algebraic topology, Academic Press, New York (in preparation).

17. J. Milnor, On spaces having the homotopy type of a CW-complex, Trans. Amer. Math. Soc. 90 (1959), 272-280.

18. R. Schon, Fibrations over a CWh-base, Proc. Amer. Math. Soc. 62 (1977), 165-166.

19. J. D. Stasheff, A classification theorem for fibre spaces, Topology 2 (1963), 239-246.

20. D. Sullivan, The genetics of homotopy theory and the Adams conjecture, Ann. of Math. (2) 100 (1974), 1-79.

Department of Mathematics, The University of Chicago, Chicago, Illinois 60637 